\title{
Distributed glycine in comet 67P/Churyumov-Gerasimenko (Corrigendum)
}

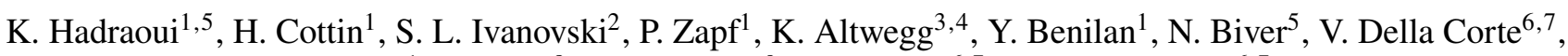 \\ N. Fray ${ }^{1}$, J. Lasue ${ }^{8}$, S. Merouane ${ }^{9}$, A. Rotundi ${ }^{6,7}$, and V. Zakharov ${ }^{6,7}$ \\ ${ }^{1}$ Laboratoire Interuniversitaire des Systèmes Atmosphériques (LISA), UMR CNRS 7583, Université Paris Diderot and Université \\ Paris Est Créteil, Créteil, France \\ e-mail: khadraoui@lisa.ipsl.fr \\ 2 INAF - Osservatorio Astronomico di Trieste, Via Tiepolo 11, 34143 Trieste, Italy \\ ${ }^{3}$ Physikalisches Institut, University of Bern, Sidlerstrasse 5, 3012 Bern, Switzerland \\ ${ }^{4}$ Center for Space and Habitability, University of Bern, Sidlerstrasse 5, 3012 Bern, Switzerland \\ ${ }^{5}$ LESIA, Observatoire de Paris, Université PSL, CNRS, Sorbonne Université, Univ. Paris Diderot, Sorbonne Paris Cité, \\ 5 place Jules Janssen, 92195 Meudon, France \\ ${ }^{6}$ INAF - Istituto di Astrofisica e Planetologia Spaziali, Ricerca Tor Vergata, Via Fosso del Cavaliere 100, 00133 Rome, Italy \\ 7 Università degli Studi di Napoli Parthenope, Dip. di Scienze e Tecnologie, CDN IC4, 80143 Naples, Italy \\ ${ }^{8}$ IRAP, Université de Toulouse, CNRS, UPS, Toulouse, France \\ 9 Max-Planck-Institut für Sonnensystemforschung, Justus-von-Liebig-Weg 3, 37077, Göttingen, Germany
}

A\&A, 630, A32 (2019), https://doi .org/10.1051/0004-6361/201935018

Key words. comets: individual: 67P/Churyumov-Gerasimenko - astrochemistry - errata, addenda

Equations (5) and (6) in the published version were incorrectly written. In addition to that, the units given in the first sentence below Eqs. (4) and (5) were incorrect. The correct versions are given below. However, the results presented in the paper were computed with the correct formulas. The figures, results, and discussion are therefore not affected.

Equation (5) should read:

$$
\begin{aligned}
\frac{\mathrm{d} n_{\mathrm{Gly}}^{\mathrm{Gas}} r^{2}}{\mathrm{~d} r}= & \frac{Q_{\mathrm{Grains}}}{v_{\mathrm{p}} v_{\text {gas }}}\left(\frac{3 M_{\mathrm{x}}}{4 \pi \rho_{\mathrm{x}} N_{\mathrm{A}}}\right)^{2 / 3} \frac{\alpha P_{\mathrm{x}} N_{\mathrm{A}}}{\sqrt{2 \pi M_{\mathrm{x}} R T_{\mathrm{p}}}}\left(n_{\mathrm{Gly}}^{\mathrm{Gr}}\right)^{2 / 3} \\
& -\frac{J_{\mathrm{Gly}}}{v_{\text {gas }}} n_{\mathrm{Gly}}^{\mathrm{Gas}} r^{2} .
\end{aligned}
$$

In Sect. 2.1, the text related to Eqs. (4) and (5) should read: Equation (4) is related to the decrease in the number of glycine molecules in a dust particule, and $n_{\text {Gly }}^{\mathrm{Gr}}$ (molec) is the total number of glycine molecules in a dust particle at a nucleocentric distance $r$. Additionally, Eq. (5) is related to the glycine density profile; $n_{\mathrm{Gly}}^{\mathrm{Gas}}\left(\mathrm{molec} \mathrm{m}^{-3}\right)$ is the volumic density of gaseous glycine at a nucleocentric distance $r$.

Equation (6) should read:

$$
R_{\beta}=\left(\beta \frac{\rho_{\mathrm{p}}}{\rho_{\mathrm{x}}}\right)^{1 / 3} R_{0} .
$$

